Supporting Information for:

\title{
Double-Vacancy Controlled Friction on Graphene: The Enhancement of Atomic Pinning
}

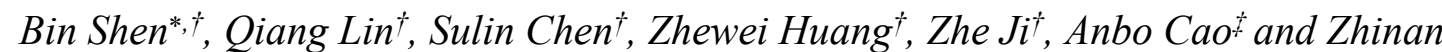

$$
\text { Zhang }{ }^{\dagger}
$$

†School of Mechanical Engineering, Shanghai Jiao Tong University, Shanghai,

China, 200240

${ }^{\star}$ SJTU-Paris Tech Elite Institute of Technology, Shanghai Jiao Tong University,

Shanghai, China, 200240

* Corresponding author. E-mail: binshen@sjtu.edu.cn (Bin Shen) 

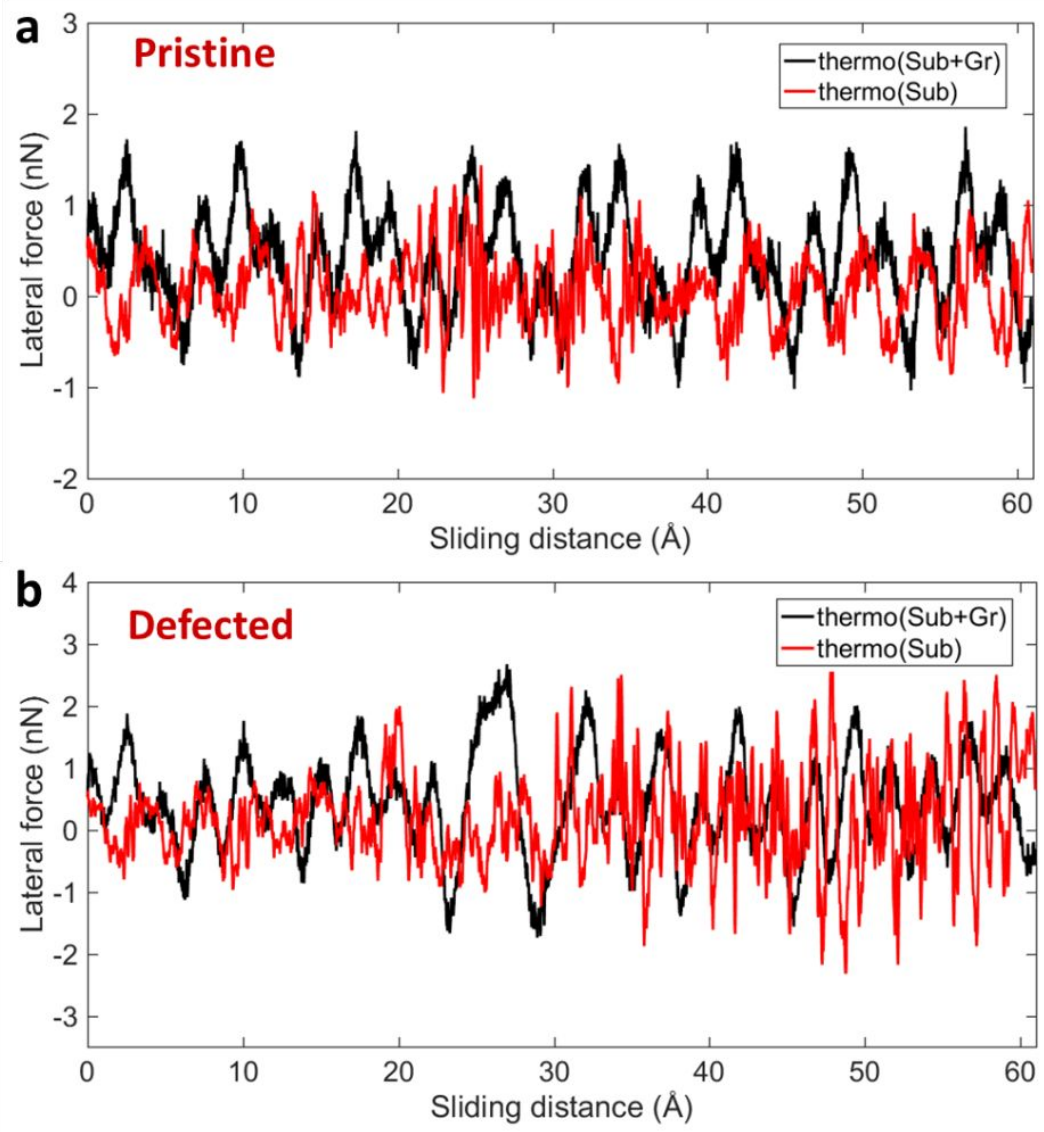

Figure S1. Lateral force versus sliding distance with the thermostat applied only to the substrate $(\mathrm{Sub})$ and both the substrate and graphene $(\mathrm{Sub}+\mathrm{Gr})$ at the normal load of $100 \mathrm{nN}$ : (a) pristine graphene; (b) graphene with the double-vacancy defect. 

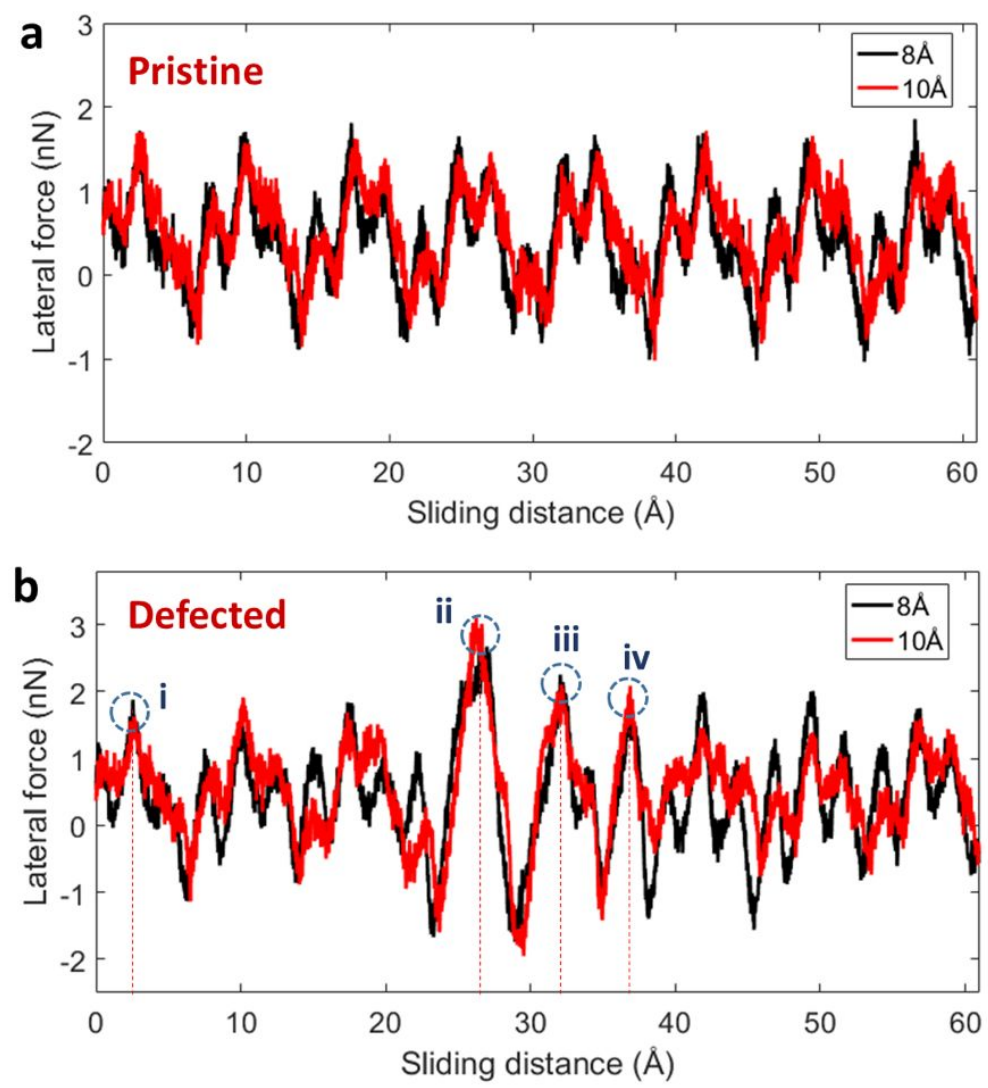

Figure S2. Lateral force curves with the cutoff radius of $8 \AA$ and $10 \AA$ at the normal load of $100 \mathrm{nN}$ : (a) pristine graphene; (b) graphene with the double-vacancy defect.

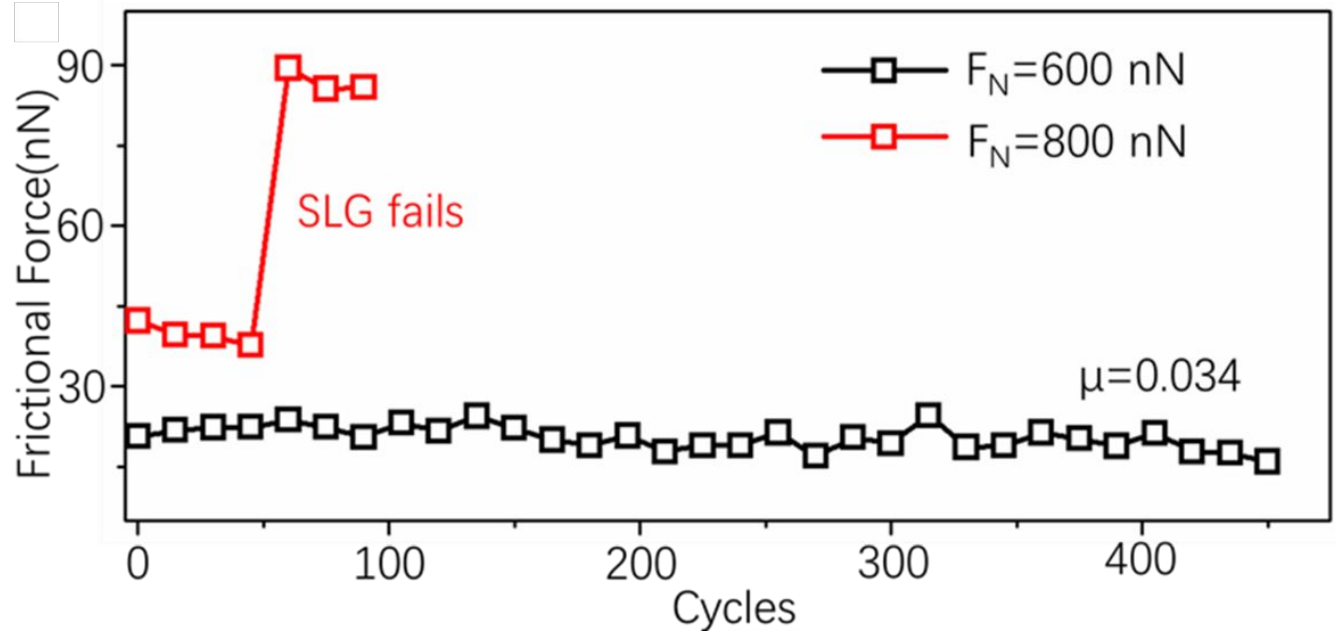

Figure S3. Frictional curves versus the sliding cycle obtained for an Si tip with a radius of $10 \mathrm{~nm}$ (Bruker, SNL-10) sliding over a SLG sheet that is transferred on an ultrasmooth diamond substrate $\left(R_{\mathrm{a}} \sim 0.314 \mathrm{~nm}\right)$ under the normal load of $600 \mathrm{nN}$ and 800 
$\mathrm{nN}$.

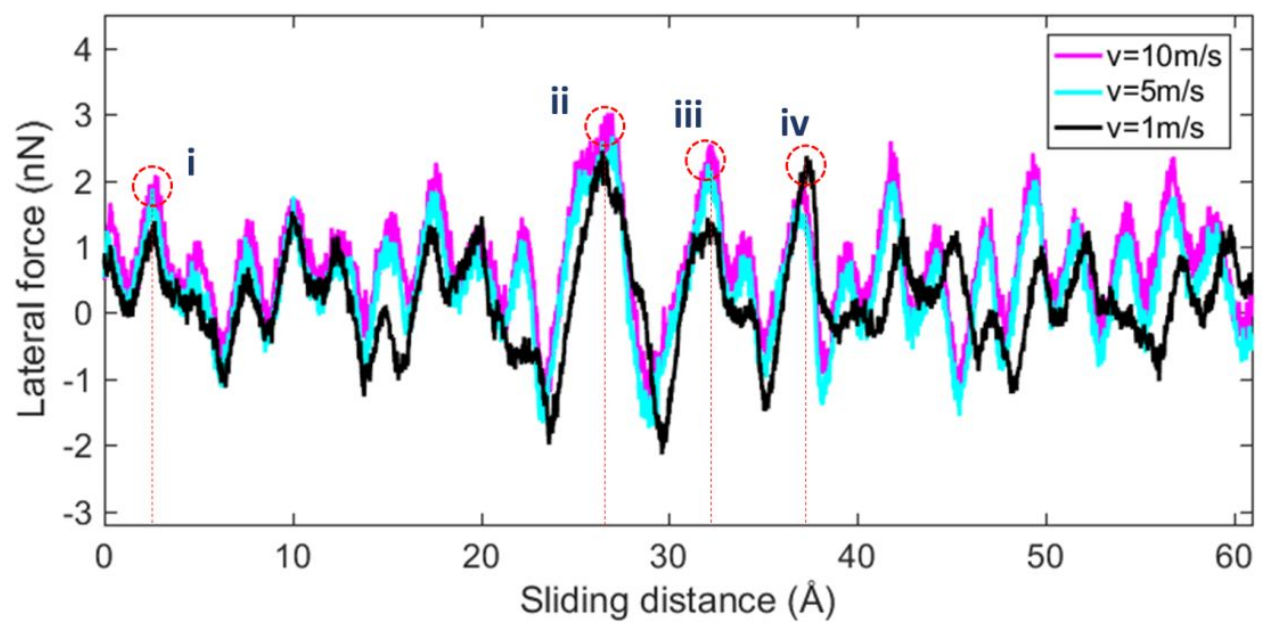

Figure S4. Lateral forces of the defected graphene sample at sliding velocities of 1-10 $\mathrm{m} / \mathrm{s}$ at the normal load of $100 \mathrm{nN}$.

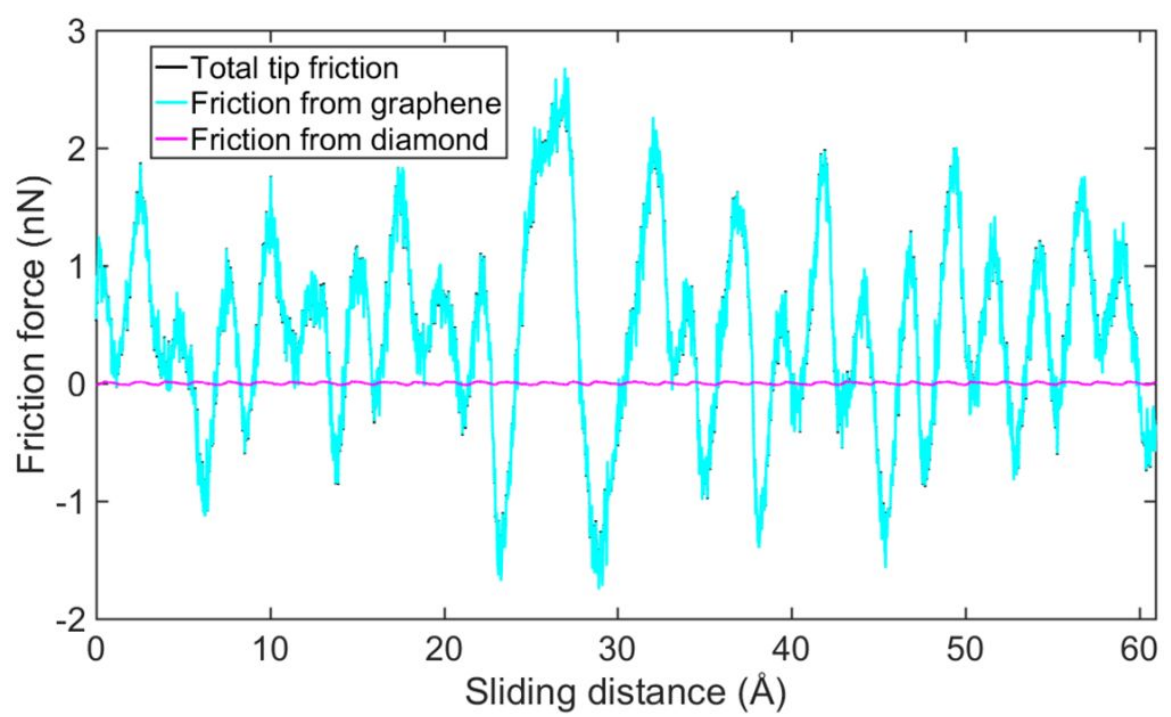

Figure S5. Comparison of friction contribution to the silicon tip from the graphene layer and the diamond substrate. 


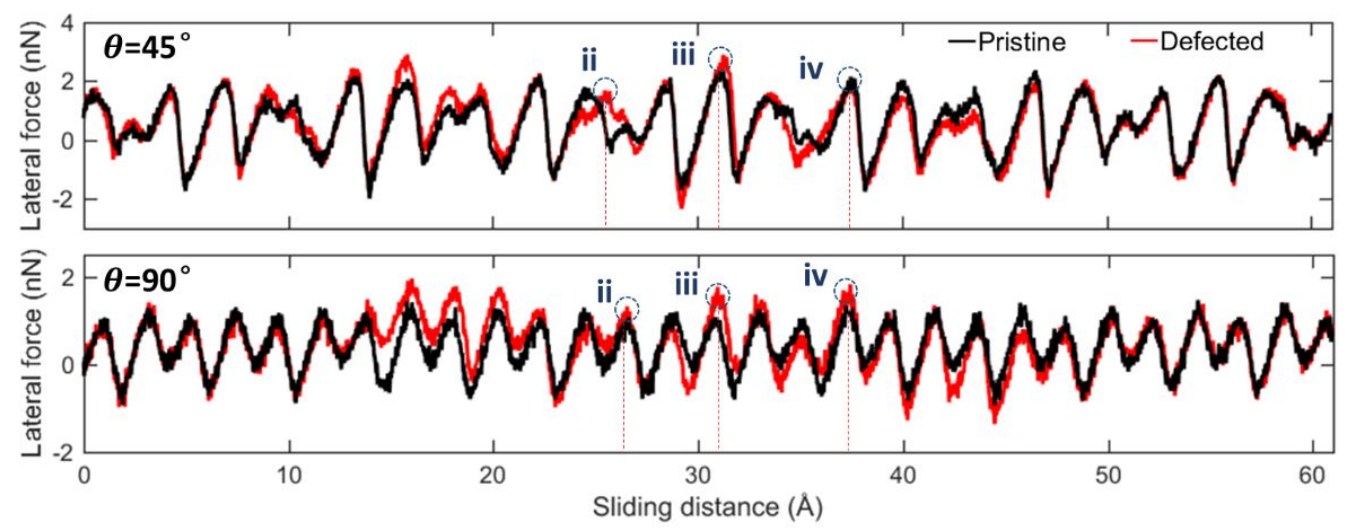

Figure S6. Lateral force curves for both the pristine and defected graphene sample at the normal load of $0 \mathrm{nN}$ for the sliding direction of $\theta=45^{\circ}$ and $\theta=90^{\circ}$. 